\title{
Min-Max decoding for non binary LDPC codes
}

\section{Dan Feng Zhao ${ }^{1, a}$ Mengjiahui ${ }^{2, b}$ Tian $\mathrm{Hai}^{3, \mathrm{c}^{*}}$}

${ }^{1,2,3}$ School of information and communication engineering, Harbin Engineering University

azhaodanfeng@hrbeu.edu.cn ${ }^{\mathrm{b}}$ mengjiahui@hrbeu.edu.cn ${ }^{c}$ mengjiahui@hrbeu.edu.cn

Key Words: Min-Max; non binary; LDPC codes; decoding; algorithm

\begin{abstract}
. with the increase in requirements on fiber-optical communication system by people, transmission quantity has been increased. In order to guarantee quick and effective fiber-optical communication, people have to use LDPC codes to implement correction modulation, of which, the ability of non-binary LDPC codes to correct abrupt error and random error become more stronger, and it is corresponds with high-order modulation, it is suitable to be used in optical transmission system with super speed and long distance, so it has become to the key point researched by people. This paper starts from non-binary LDPC codes to analyze the principle application of correcting matrix and Tanner diagram, it also illustrates coding and decoding of LDCP codes and puts forward Min-Max non binary LDPC codes algorithm, which simplifies calculation of non binary LDPC codes and can effectively promote enhancement of communication in error correction.
\end{abstract}

\section{Introduction}

As the soft decision technology universally researched by people, LDPC has excellent ability in error correction; error level is low and can be concurrently realized. Meanwhile, research on binary LDPC codes is relatively mature, including binary LDPC codes and decoding algorithm, capacity analysis method, search algorithm of optimal degree based on Gaussian white noise channel and Rayleigh fading channel with irregular code. However, compare non-binary LDPC code with LDPC codes, its ability in abrupt error correction and random error correction is stronger, and it is more suitable with high-order modulation, so it is suitable to be used in optical transmission system with super speed and long distance.

\section{Overview on non-binary LDPC codes}

LDPC codes was put forward by Gallager in 1960, it is the linear block code, ix represents better capacity in data transmission and data storage, it is exclusively determined by generation matrix $G$ or parity check matrix $\mathrm{H}$, so it can define LDPC codes by parity check matrix $\mathrm{H}$. Of which, $\mathrm{H}$ has 4 natures, which means each line has $\mathrm{p} 1$, each kind has $\mathrm{r}$ and 1 , the position between any 2 lines is the same and the number of valuing 1 dose not exceed 1 , the line number comparison between $p, t$ as well as code length and $\mathrm{H}$ is very small. From these natures we can see that parity check matrix and $H$ respectively has special line weight $p$ and parallel weight $r$, and any 2 lines have 1 exceed the same position, the density of 1 is very small, so it is called as parity check matrix with low density, the details are indicated by the following figure 1 :

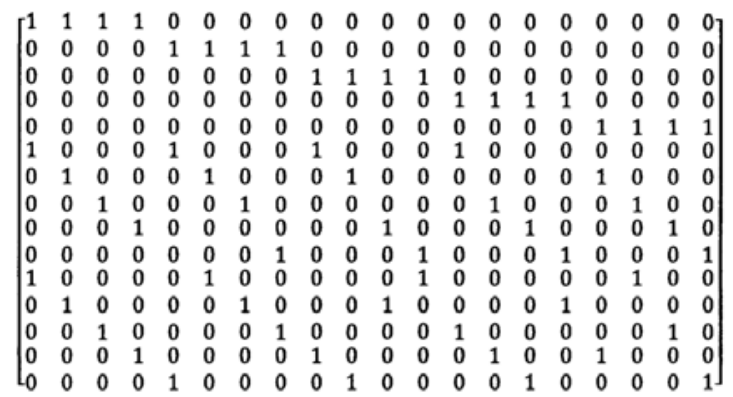

Figure 1 Parity check matrix with low=density of LDPC

Except to use check matrix to express LDPC codes, it can also use bidirectional graph model to 
indicate and describe, of which, Tanner graph is universally used. Check matrix $\mathrm{H}_{\mathrm{M} \times \mathrm{N}}$ designates linear block code with code length of $\mathrm{N}$, Tanner graph is composed of 2 nodes assemble; they are respectively $\mathrm{v}_{\mathrm{b}}$, vc. $\mathrm{v}_{\mathrm{b}}$ is composed of nodes represent $\mathrm{N}$ code word bit, it is marked as $\mathrm{V}_{0}, \mathrm{~V}_{1}, \ldots$, $\mathrm{V}_{\mathrm{N}-1}$, which is variable node or bit node, it is corresponds to rank of check matrix. $\mathrm{v}_{\mathrm{c}}$ is composed of nodes with $\mathrm{M}$ check or check equation, which is marked as $\mathrm{c}_{\mathrm{o}}, \mathrm{C}_{1}, \ldots, \mathrm{C}_{\mathrm{M}-1}$, it is called as check node, it is corresponds to row of check matrix. By combining with parity check matrix of LDPC codes, we can see that it dose not exist 2 code elements bit are tested by 2 different check nods at the same time, so in Tanner graph, one LDPC code excludes ring with length of 4.

Suppose parity matrix of one $(8,4)$ linear block code is as follows:

$$
H=\left[\begin{array}{llllllll}
1 & 1 & 0 & 0 & 0 & 0 & 1 & 0 \\
0 & 0 & 1 & 1 & 1 & 0 & 0 & 0 \\
0 & 0 & 0 & 1 & 0 & 1 & 0 & 1 \\
1 & 0 & 1 & 0 & 0 & 1 & 1 & 0
\end{array}\right]
$$

Figure $2(8,4)$ Parity check matrix diagram

The corresponding Tanner graph includes 8 variable nodes, they are respectively $V_{1}, V_{2}, V_{3}$, $\mathrm{V}_{4}, \mathrm{~V}_{5}, \mathrm{~V}_{6}, \mathrm{~V}_{7}, \mathrm{~V}_{8}, 4$ check nodes are respectively $\mathrm{c}_{1}, \mathrm{C}_{2}, \mathrm{c}_{3}, \mathrm{c}_{4}$, they are connected by side, the obtained route, one is node order $\left(\mathrm{v}_{1}, \mathrm{C}_{1}, \mathrm{v}_{7}, \mathrm{c}_{4}, \mathrm{v}_{1}\right)$, it is one ring with length of 4 , the other node order is $\left(\mathrm{V}_{3}, \mathrm{C}_{2}, \mathrm{v}_{4}, \mathrm{C}_{3}, \mathrm{v}_{6}, \mathrm{c}_{4}, \mathrm{v}_{3}\right)$, it is one ring with length of 6 , which is indicated by figure 3:
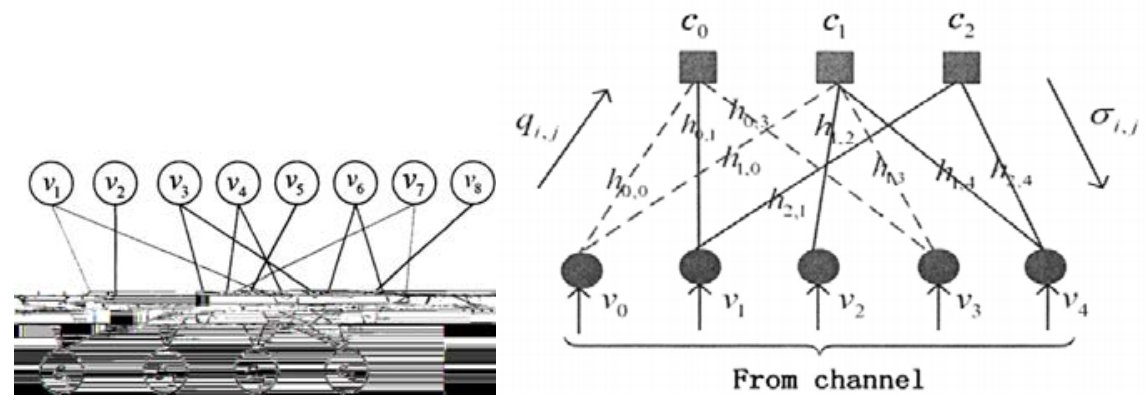

Figure 3Tanner diagram of $(8,4)$ linear block node Figure 5 Tanner graphs of non binary LDPC codes

In 1998, Davey and Mackay put forward codes based on GF (q) field, from the theory result, it uses non-binary LDPC code, which can obtain big gain in proper length, under condition of low code ratio from $1 / 4$ to $1 / 2$, the ability of error correction is better than binary LDPC code with the same code ratio., Therefore, as for non binary LDPC codes, it can be regarded as the popularization of binary LDPC code in finite field, and one linear block code, which can be used to check matrix and describe Tanner graph.

Of course, check matrix of non binary LDPC code and binary LDPC code is different. Check matrix $\mathrm{H}_{\mathrm{m} \times \mathrm{n}}$ of non binary LDPC, $\mathrm{m}$ is line number, $\mathrm{n}$ is row number, so every element number $\mathrm{h}_{\mathrm{i}}$, $\mathrm{j}(0 \leq \mathrm{i}<\mathrm{m}, 0 \leq \mathrm{j}<\mathrm{n})$ of matrix $\mathrm{H}$ all are taken from finite field $\mathrm{GF}(\mathrm{q})$, the number of non-zero element in each row is regarded as row weight. When the non-zero element number of every line or every row in matrix is the same, which is regular non-binary LDPC code, otherwise is irregular non-binary LDPC code, check matrix $\mathrm{H}(3,7)$ of LDPC codes on GF $\left(\mathrm{q}=2^{3}\right)$ is as follows:

$$
H=\left[\begin{array}{lllllll}
2 & 6 & 1 & 0 & 3 & 0 & 0 \\
0 & 2 & 4 & 6 & 0 & 2 & 0 \\
3 & 0 & 2 & 7 & 0 & 0 & 5
\end{array}\right]
$$

Figure 4 LDPC check node of $\mathrm{H}(3,7)$ 
From the above-mentioned matrix, we can see that line weight is 4 , the maximum row weight is 3 , code better $\mathrm{c}=\left(\mathrm{c}_{1}, \mathrm{c}_{2}, \mathrm{c}_{3}, \mathrm{c}_{4}, \mathrm{c}_{5}, \mathrm{c}_{6}, \mathrm{c}_{7}\right)$, its corresponding check equation is as follows:

$$
\begin{aligned}
& 2 \mathrm{c}_{1}+6 \mathrm{c}_{2}+\mathrm{c}_{3}+3 \mathrm{c}_{5}=0 \\
& 2 \mathrm{c}_{2}+4 \mathrm{c}_{3}+6 \mathrm{c}_{4}+2 \mathrm{c}_{6}=0 \\
& 3 \mathrm{c}_{1}+2 \mathrm{c}_{3}+7 \mathrm{c}_{4}+5 \mathrm{c}_{7}=0
\end{aligned}
$$

Tannner diagram is one bidirectional graph; its diagram is as follows:

In the above figure, check node, variable node and loop connected by beginning and end is regarded as cycle, the sides number included by cycle is called as cycle length, the cycle length of the shortest cycle in Tanner graph is called as girth of LDPC codes.

\section{Coding and decoding of LDPC codes}

Generally speaking, on using information channel to convey information, in order to guarantee reliability and transmission efficiency of information, it needs to use certain algorithm to realize coding and decoding. Since Shannon coding theory in 1948, people all use this as basis on coding research. When it meets 3 conditions, the ability of Shannon theory is very good, they are respectively as follows: code uses random coding way, the length of coding should be enough long and approach length is the most ideal. On decoding, it uses maximum likelihood ratio decoding(Max. LLr). As for LDPC codes parity check with low density, it needs to use intersection in the coding process, so the code word after coding has random character, it needs iteration of many times in the decoding process, so it is relatively close to Shannon. For example, information source is $\mathrm{u}$, parity check matrix $\mathrm{H}$ is $\mathrm{M} \times \mathrm{N}$, the code word through coding $\mathrm{V} v=[\mathrm{c} \mid \mathrm{u}]$, c is check information, it divides parity check matrix into when $A$ is $M \times N$ matrix, $B$ is matrix of $M \times(N-M)$, because $\mathrm{v} \cdot H^{T}=0$, so it can get the following:

$$
\left[\begin{array}{ll}
\mathrm{c} & \mathrm{u}
\end{array}\right]\left[\begin{array}{l}
A^{T} \\
B^{T}
\end{array}\right]=0 \rightarrow \mathrm{c} \cdot A^{T}+\mathrm{u} \cdot B^{T}=0 \rightarrow \mathrm{c}=\mathrm{u} \cdot B^{T}\left(A^{T}\right)^{-1}
$$

Only A is invertible matrix, it can get check information, so it can complete coding process. It makes line exchange with matrix $\mathrm{H}$. It can be explained as position of exchanging check nodes in Tanner graph, coding result has not been affected, on making line and row exchange, it just rearranges code word order of coding, and it will make revertible exchange according to the original sequence after coding is completed. Of course, when corresponding matrix A of parity matrix $\mathrm{H}$ is revertible, it can directly use way of quick coding to complete information input and make coding.

\section{Decoding algorithm of Min-max non-binary LDPC coeds}

In order to further simplify algorithm of non-binary LDPC decoding, this paper puts forward one kind of calculation algorithm with high efficiency, which is Min-max decoding algorithm, it has incomparable advantages such as low complication in decoding and needs small storage space, quick decoding speed etc. When using $\alpha$ to represent primitive element of non-binary LDPC code on GF (q), the power of a generates all the non-zero elements, while in Tanner graph of $\mathrm{H}$ matrix, when elements on the $\mathrm{i}$ line $\mathrm{j}$ row in $\mathrm{H}$ is non-zero elements, it has one straight line between the $\mathrm{i}$ node and $\mathrm{j}$ variable node, which is one side of Tanner graph, so it can transmit information and make iteration decoding on LDPC codes. While as for one received code element, it may be any one in the q elements on the transmission terminal, so every side of Tanner graph needs to transmit $\mathrm{q}$ reliability information to represent probability of every element. In log-domain Min-max algorithm, reliability information of code element can use log likelihood ratio $(L L R) L(\alpha)=\log (P(\beta) / P(\alpha))$, of which, $\beta$ is the element with the biggest probability, $\alpha$ is any one element. Its Min-max decoding algorithm is as follows: 
Initialization:

$$
\mathrm{u}_{\mathrm{m}, \mathrm{n}}(\alpha)=\mathrm{r}_{\mathrm{n}}(\alpha)
$$

Iteration:

Process check node: $\mathrm{v}_{\mathrm{m}, \mathrm{n}}(\alpha)=\min _{\left(\alpha_{\mathrm{j}}\right) \in L\left(\mathrm{~m} \mid \alpha_{\mathrm{n}}=\alpha\right)}\left(\max _{\mathrm{j} \in \mathrm{S}_{\mathrm{v}}(\mathrm{m}) \backslash \mathrm{n}} \mathrm{u}_{\mathrm{m}, \mathrm{j}_{\mathrm{j}}}\left(\alpha_{\mathrm{j}}\right)\right)$

$\left.\begin{array}{c}\mathrm{u}_{\mathrm{m}, \mathrm{n}}^{\prime}(\alpha)=\mathrm{r}_{\mathrm{n}}(\alpha)+\sum_{\mathrm{i} \in S_{\mathrm{c}}(\mathrm{n}) \backslash \mathrm{m}} \mathrm{v}_{\mathrm{i}, \mathrm{n}}(\alpha) \\ \text { Process variable node: } \\ \mathrm{u}_{\mathrm{m}, \mathrm{n}}(\alpha)=\mathrm{u}_{\mathrm{m}, \mathrm{n}}^{\prime}(\alpha)-\min _{\mathrm{w} \in G F(\mathrm{q})}\left(\mathrm{u}_{\mathrm{m}, \mathrm{n}}^{\prime}(\mathrm{w})\right)\end{array}\right\}$

Posterior information calculation: $\tilde{\mathrm{r}}_{\mathrm{n}}(\alpha)=\mathrm{r}_{\mathrm{n}}(\alpha)+\sum_{\mathrm{i} \in S_{\mathrm{c}}(\mathrm{n})} \mathrm{v}_{\mathrm{i},{ }_{\mathrm{n}}}(\alpha)$

On processing of check node, it directly calculates complication of $L\left(\mathrm{~m} \mid \alpha_{\mathrm{n}}=\alpha\right)$ is very higher, proposal of forward-backward algorithm avoids direct calculation and simplifies decoding process, it uses $n_{i}$ to express the $i$ variable node connected to check node, and $d_{c}$ to express degree of check node, the steps of forward-backward are as follows:

Forward calculation:

$$
\left.\begin{array}{c}
\mathrm{f}_{1}(\alpha)=\mathrm{u}_{\mathrm{m}, \mathrm{n}_{1}}(\alpha) \\
\mathrm{f}_{\mathrm{i}}(\alpha)=\min _{1<\mathrm{i}<\mathrm{d}_{\mathrm{c}}}\left(\max \left(\mathrm{f}_{\mathrm{i}-1}\left(\alpha^{\prime}\right), \mathrm{u}_{\mathrm{m}, \mathrm{n}_{\mathrm{i}}}\left(\alpha^{\prime \prime}\right)\right)\right)
\end{array}\right\}
$$

Backward calculation:

$$
\left.\begin{array}{c}
\mathrm{b}_{\mathrm{d}_{\mathrm{c}}}(\alpha)=\mathrm{u}_{\mathrm{m}, \mathrm{n}_{\mathrm{d}_{\mathrm{c}}}}(\alpha) \\
\mathrm{b}_{\mathrm{i}}(\alpha)=\min _{1<\mathrm{i}<\mathrm{d}_{\mathrm{c}}}\left(\max \left(\mathrm{b}_{\mathrm{i}+1}\left(\alpha^{\prime}\right), \mathrm{u}_{\mathrm{m}, \mathrm{n}_{\mathrm{i}}}\left(\alpha^{\prime \prime}\right)\right)\right)
\end{array}\right\}
$$

Calculation of check node information:

$$
\left.\begin{array}{c}
\mathrm{v}_{\mathrm{m}, \mathrm{n}_{1}}(\alpha)=\mathrm{b}_{2}(\alpha) \\
\mathrm{v}_{\mathrm{m}, \mathrm{n}_{\mathrm{d}_{\mathrm{c}}}}(\alpha)=\mathrm{f}_{\mathrm{d}_{\mathrm{d}}-1}(\alpha) \\
\min _{\alpha^{\prime \prime}=\alpha}\left(\max \left(\mathrm{f}_{\mathrm{i}-1}\left(\alpha^{\prime}\right), \mathrm{b}_{\mathrm{i}+1}\left(\alpha^{\prime \prime}\right)\right)\right)
\end{array}\right\}
$$

This kind of Min-max non-binary LDPC decoding algorithm has greatly optimized the traditional calculation; it simplifies calculation and has obvious advantage.

\section{Simulation and performance analysis of non binary LDPC code modulation}

The simulation system here is 16 QAM modulation and AWGN channel component code is based on GF (22) the minimum column weight of 3 NB - LDPC code, decoding the 20 choice encoding scheme, the main effect of different code length and other coding modulation schemes comparison from two aspects of LDPC code the error correcting performance with SE.

Firstly, the different impact on the programme of study, different code length $\mathrm{R}$ component code rate was 0.8 based on GF (22) LDPC (102208176), LDPC (51004098), LDPC (25402032) were studied, the simulation is shown in figure 6: 


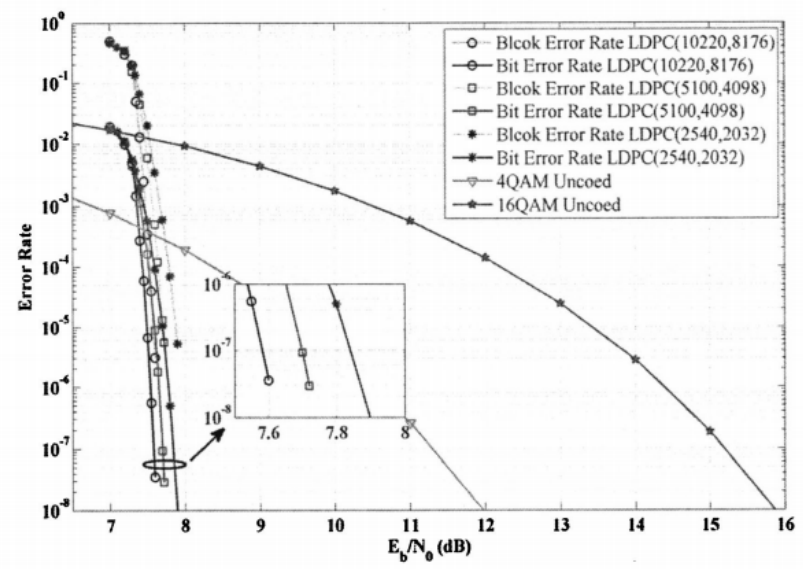

Fig. 6 Comparison of LDPC - NB coded modulation schemes under different codes

The solid and dashed lines represent the bit error rate and packet error rate, from the figure can know simulation scheme in any code length under BER are far lower than the 4-QAM modulation scheme based on non encoding, and SE is a 3.2 bit / symbol, not higher than 4-QAM modulation scheme encoding SE. Visible, whether it is BER or spectrum utilization, the advantages of non binary LDPC coded modulation scheme are more obvious.

Compared with the non coding 16-QAM modulation scheme, although SE is not high, but in the bit error rate, the 16.QAM modulation scheme is not encoded very far. At the same time, it is also seen that simply improve the modulation order, but a system reliability in exchange for SE technology, this shows that the higher the modulation order, the worse the reliability. Therefore, even if the SE is high, it does not apply to demanding optical communication systems.

From each performance curve, the error correcting performance was better than the performance of solid line, means that no matter how much is in the same code length, code parameters, BER is always lower than the packet error rate. The LDPC (102208176) performance is better than that of LDPC (51004098) LDPC (51004098) of the performance, the performance is better than the performance of LDPC (25402032), and in the same BER, code length, the higher the NCG, the function of data are shown in table 1.

Table 1 Comparison of NB-LDPC coded modulation schemes under different codes

\begin{tabular}{|l|l|l|}
\hline Code Type & Code Rate & NCG at BER of 10-7/dB \\
\hline $\operatorname{LDPC}(10220,8176)$ & 0.8 & 7.62 \\
\hline $\operatorname{LDPC}(5100,4098)$ & 0.8 & 7.5 \\
\hline $\operatorname{LDPC}(2540,2032)$ & 0.8 & 7.35 \\
\hline
\end{tabular}

Simulation results show that, under the same transmission power, the length of the code is longer, the lower the BER. In the same BER requirements, the length of the packet is longer, the smaller the required $\mathrm{Eb} / \mathrm{N} 0$.

\section{Conclusion}

In a word, in the development process of optical communication development and channel coding, research on non binary coding and block code decoding is always in deepening development. In actual application, the ability in abrupt error correction and random error of non binary LDPC code becomes stronger, and it is suitable with high-order modulation, it is more suitable to be used in optical transmission system with super speed and long distance. In order to further enhance application of non binary LDPC codes, it needs to continually optimize its decoding algorithm, Min-max algorithm effectively simplifies non binary LDPC codes and it has huge application, practice values. 


\section{References}

[1]Liu Jixin. Discussion on Application of Non binary LDPC Codes Modulation in Optical-fiber Communication [J]. Heilongjiang Science and Technology Information，2013（20）: 169

[2]Yang Wei, Zhang Wei. Multi-binary LDPC Codes Algorithm Based on Layered Decoding and Min-max [J]. Journal of Electronics and Information, 2013,35 (7) : 1677-1681

[3]He Kai, Design Research on Non-binary LDPC Codes Demodifier [D]. Master's degree thesis of Nanjing University, 2012

[4]Yu Qian. Research on Non-binary LDPC Codes in High-speed Optical Transmission System [D]. Master's degree thesis of Beijing University of Posts and Telecommunications, 2015

[5]L Yang, F Liu, H Li.Min-Max Decoding for Non-Binary LDPC Codes [J]. Springer Berlin Heidelberg, 2013 (210) : 125-134

[6]Qiang Longfei. Research on NB-QC-LDPC in Non-linear Optical-fiber Communication [D]. Master's degree thesis of Beijing Jiaotong University, 2015

[7]He Li. Research on Modulation Based on Non-binary LDPC Codes in Optical Transmission System [D]. Master's degree thesis of Chongqing University of Posts and Telecommunications, 2013

[8]Wang Haiping. Research on FEC of the Third Generation in Optical-fiber Communication System and Application of NB-LDPC Codes in WDM-PON [D]. Master's degree thesis of Zhejiang University, 2014 